\title{
Platelets Indices and Different Germs of Neonatal Sepsis
}

\author{
Razieh Sangsari ${ }^{1,{ }^{*}}$, Maliheh Kadivar ${ }^{1}$, Rasoul Fardi ${ }^{2}$, Saeid Jedary Attary ${ }^{1}$ and Ali Jafari ${ }^{2}$ \\ ${ }^{1}$ Department of Pediatrics, Tehran University of Medical Sciences, Tehran, Iran \\ ${ }^{2}$ Children's Medical Center, Pediatrics Center of Excellence, Tehran, Iran \\ "Corresponding author: Department of Pediatrics, Tehran University of Medical Sciences, Tehran, Iran. Tel: +98-2166920983, Fax: +98-2166929235, Email: raz3532@yahoo.com
}

Received 2018 June 13; Revised 2018 December 02; Accepted 2018 December 07.

\begin{abstract}
Background: Due to high incidence of sepsis as the main cause of neonatal mortality, early detection and proper treatment are important in reducing of neonatal mortality. Therefore, researchers are looking for a way of early diagnosis and treatment of sepsis. Platelet indices are one of these ways. But, can they determine different causing germs of neonatal sepsis?

Methods: In this study, the septic neonates with positive blood culture were studied. Neonates with positive blood culture were divided into three groups; Gram-positive, Gram-negative and fungal infections. Platelet count and other parameters of platelets including PDW (platelet distribution width), and MPV (mean platelet volume) were evaluated, and compared with respect to different germs of septic neonates.

Results: In this study 99 newborns with positive blood cultures were evaluated. Of these 13 neonates were excluded because of contaminated culture. In the remaining 86 cases thrombocytopenia, thrombocytosis, PDW, and MPV in different types of neonatal sepsis were evaluated and compared.

Conclusions: Although thrombocytosis may be a laboratory finding for sepsis but it is not common in early onset sepsis. Elevated PDW is more common in Gram-negative infections and may be helpful in determining different germs of neonatal sepsis but mean values of PDW in Gram-negative infections are not more than that caused by other germs.
\end{abstract}

Keywords: Neonatal Sepsis, Blood Culture, Platelets

\section{Background}

Neonatal sepsis is a systemic infection in neonatal period. It is still a major cause of morbidity and mortality of newborns, especially in developing countries (1). Early diagnosis and treatment is essential to reduce morbidity and mortality (2). For this purpose some hematological findings can be helpful as blood culture is not available before 48 - 72 hours. Platelet indices; platelet count, PDW (platelet distribution width), and MPV (mean platelet volume) belong to this category of findings. Platelet count is important and thrombocytopenia is an early finding in neonatal sepsis (3). Although thrombocytopenia is seen in different types of neonatal sepsis it is reported to be more common in infections caused by Gram negative germs $(2,4)$ specially Klebsiella spp (5) 'Enterobacter spp.' (6) and fungal infections (3, 7). Some articles showed elevated PDW and MPV in septic neonates (3) and significant elevated level of MPV and even late return to normal level after treatment in Coagulase-negative staphylococci (8). If this becomes proven, regarding that the results of blood culture are available after 48 hours, this parameters can help to come to an early diagnosis of sepsis and even recognize the type of germs causing it (9). So this can be a clue for proper treatment.

\section{Objectives}

In this study, we evaluated platelet count, PDW, and MPV in neonatal sepsis caused by different types of germs. Comparing these findings the importance of them in early detection of the types of sepsis germs must be recognized.

\section{Methods}

In a prospective sectional study from September 2016 to March 2017, the septic neonates with positive blood culture who were referred to the Children's Medical Center Hospital of Tehran were studied.

The neonates included in the study had at least one positive blood culture and two or more of the following symptoms: fever that would not drop with hydration of the patient, poor feeding, lethargy, irritability, tachypnea, and tachycardia. Excluded neonates were those without signs of infection, and a positive blood culture of a suspected 
contamination of sample and neonates with platelet disorders other than sepsis.

Blood culture was performed in $1 \mathrm{cc}$ peripheral vessel sample in BACTEC medium with agar diffusion method. Coulter counters method was used for CBC (complete blood count). Platelets less than 150000 per microliter were considered as thrombocytopenia and more than 450000 as thrombocytosis. MPV up to 10 femtoliter was considered as being in normal limits and more than that as elevated. PDW higher than $17 \%$ was considered as significant.

After assessment the types of sepsis germs in positive blood cultures of the neonates, the ratios of thrombocytopenia, thrombocytosis, high MPV, and PDW in different germs were evaluated. Chi-squared test was used for comparison of qualitative variables. For comparison of quantitative variables, $t$-test, paired $t$-test, Fisher exact test and One-way analysis of variance (ANOVA) were applied. Normal distribution was calculated using KolmogorovSmirnov test. P value less than 0.05 was taken as significant.

The platelet indices measured were compared in different types of neonatal sepsis.

\section{Results}

In this study, 99 neonates admitted to Children's Medical Center Hospital with positive blood culture were studied. Of these, 13 neonates were excluded as contaminated.

15 cases had early onset, 36 late onset sepsis and 35 nosocomial infection. The most common cause of blood infection in studied newborns was Gram-positive Staphylococcus epidermidis (22 cases, $25.5 \%$ ).

Thrombocytosis was seen in $15.2 \%$, thrombocytopenia in 36.4\%, elevated MPV in 25.6\% and elevated PDW in $24.3 \%$ of included neonates. Thrombocytosis seen in $0 \%$ of early onset, $40 \%$ of late sepsis and $15.8 \%$ of nosocomial infections (Pvalue 0.02) which was significant, thrombocytopenia existed in $33.3 \%$ of early, $40 \%$ of late sepsis and $50 \%$ of nosocomial infections (P value 0.52). Elevated MPV was seen in $85.7 \%$ of early, $69.2 \%$ of late sepsis and $73.5 \%$ of nosocomial infections (P value 0.16 ) and elevated PDW in $28.5 \%$ of early, $18.5 \%$ of late sepsis and $27.2 \%$ of nosocomial infections (P value 0.72 ) (Table 1). The ratio of platelet index for different germs in different types of neonatal sepsis was not estimated because of small number of cases in each group (Table 2).

Thrombocytosis was seen in $34.2 \%$ of Gram-positive, $51.6 \%$ of Gram-negative and $66.6 \%$ of fungal infections (P value $=0.26)$. Thrombocytopenia seen in $28.5 \%$ of Grampositive, $16.6 \%$ of Gram-negative and $0 \%$ of fungal infections $(\mathrm{P}$ value $=0.62$ ). Elevated MPV was seen in $19.5 \%$ of
Gram-positive, 36\% of Gram-negative and 33.3\% of fungal infections ( $P$ value $=0.26$ ), elevated PDW was in $15.2 \%$ of Gram-positive, $44 \%$ of Gram-negative and $0 \%$ of fungal infections $($ P value $=0.026)$ (Table 3$)$. The mean value of PDW in Gram-negative infections is not more than that of Grampositive sepsis and fungal infections, i.e. 16.2\%, 15.3 and $14.4 \%$ respectively $(P$ value $=0.323)$.

\section{Discussion}

There are several hematological findings for early diagnosis of neonatal sepsis before availability of the blood culture results. Thrombocytopenia is one of these (3). Thrombocytopenia occurs in half of the cases with proven sepsis (10) and is used as a marker for screening of neonatal sepsis. It has been reported to be more common in association with some sepsis causing germs. This will be helpful to choose appropriate empirical therapy soon before availability of blood culture results. Ahmed et al. (11) reported to have seen thrombocytopenia more common in gram negative sepsis and that it had more prolonged duration in these cases than in Gram-positive sepsis. Some authors have even seen severe thrombocytopenia with Gramnegative sepsis (4). In addition, it correlated with the outcome of the patients especially in VLBW newborns (12). In a study Ahmad et al. (12) showed that thrombocytopenia was more common in fungal sepsis and Bhat et al. (13) reported high incidence of thrombocytopenia with Gram-negative and fungal infections. In addition prolonged thrombocytopenia, very low platelet levels, and need to platelet transfusion were poor prognostic factors. In a similar study by Alshorman Abdullah et al. thrombocytopenia was detected in $25 \%$ of infants with sepsis, but significant differences were not observed in Gram-positive and negative infections (14). All studies confirmed thrombocytopenia to be common in septic neonates, but can thrombocytopenia determine different types of neonatal sepsis germs, too?

Other platelet findings in diagnosis and predicting of sepsis are PDW and MPV (15). Elevated MPV (16) and PDW may be used as a marker for screening of sepsis. Choudhary et al. (17) reported that high MPV, high PDW and thrombocytopenia are more common in late onset than early onset sepsis. Although, Shaaban and Safwat (18) found MPV as a marker of early onset sepsis. Catal et al. (1) estimated that elevated MPV can be a diagnostic factor along with other findings and can even be utilized for following the course of the sepsis in neonates but, in line with our study, they didn't find any correlation between elevated MPV and different kinds of sepsis germs. Akarsu et al. (19) also didn't find any correlation between elevated MPV and PDW and different germs of sepsis, although we found 


\begin{tabular}{|c|c|c|c|c|c|c|c|c|}
\hline type of Infection & Thrombocytosis & PValue & Thrombocytopenia & PValue & High MPV & PValue & High PDW & P Value \\
\hline Early onset sepsis, \% & 0 & \multirow{3}{*}{0.02} & 33.3 & \multirow{3}{*}{0.52} & 85.7 & \multirow{3}{*}{0.16} & 28.5 & \multirow{3}{*}{0.72} \\
\hline Late onset sepsis, \% & 40 & & 40 & & 69.2 & & 18.5 & \\
\hline $\begin{array}{l}\text { Nosocomial infection, } \\
\%\end{array}$ & 15.8 & & 50 & & 73.5 & & 27.2 & \\
\hline
\end{tabular}

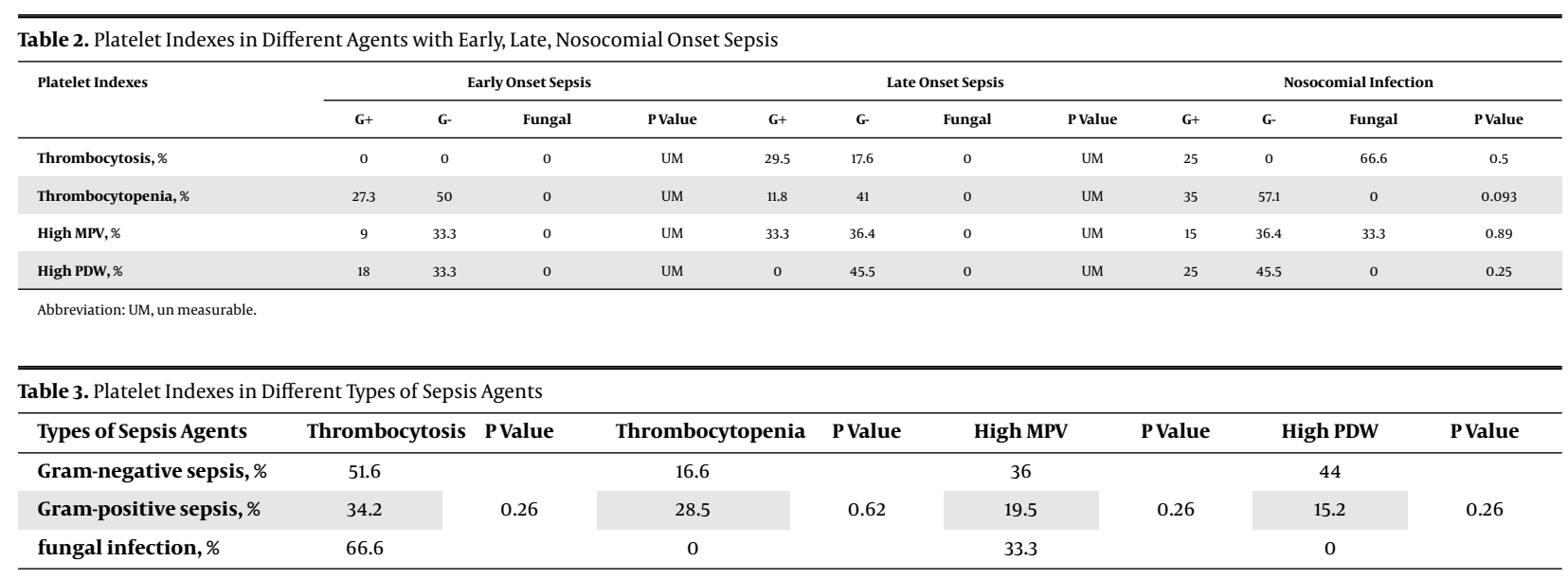

that elevated PDW is more common in Gram-negative infections $($ Pvalue $=0.026$ ). Some authorss reported that septic patients with PDW levels greater than $18 \%$ have worse outcome (20). Ahmad and Waheed (3) did not accept this and introduced thrombocytopenia instead as the predictive factor in neonatal sepsis and found higher mortality rates in thrombocytopenic neonates.

\subsection{Conclusions}

Evaluating platelet indices in neonates with different sepsis germs we found that, although thrombocytosis may be a laboratory finding in septic neonates, it is not common in early onset infections ( $P$ value $=0.028$ ). In addition, thrombocytopenia, elevated MPV or PDW can be laboratory findings for diagnosis of sepsis but are not specific for special types (namely early, late sepsis, nosocomial infection) of infection. Thrombocytosis, thrombocytopenia and elevated MPV are seen in Gram-positive, Gramnegative and fungal infections and they are not specific findings in determining different types of sepsis germs but PDW can be helpful because it was observed more common in Gram-negative infections in the present study (P value = 0.026), although the mean value of PDW in Gram-negative infections was not more than that in cases caused by other germs.

So, note that:

- In a young neonate with thrombocytosis sepsis is not the first thing to be considered because thrombocytosis is not common in early onset sepsis.
- In septic neonates pay attention to platelet indices, if PDW is over $17 \%$, Gram-negative infections are more probable than Gram-positive and fungal infections. This is important for starting empiric therapy.

We admit that we had limitations in our study. The study was carried out in out-born hospital patients, so we had only few neonates with early onset sepsis. In addition, the number of studied neonates was limited. So, multicenter studies with larger sample size are necessary to come to more reliable conclusions.

\section{Acknowledgments}

We thank the staff of Neonatal Intensive Care Unit, Neonatal Ward, and Laboratory of the Children's Medical Center Hospital, Tehran, for their valuable cooperation.

\section{Footnotes}

Authors' Contribution: Study concept and design: Maliheh Kadivar; drafting of the manuscript: Razieh Sangsari; acquisition of data: Ali Jafari; analysis and interpretation of data, statistical analysis: Rasoul Fardi; critical revision of the manuscript for important intellectual content: Razieh Sangsari; study supervision: Saeid Jedary Attary.

Conflict of Interests: It is not declared by the authors.

Ethical Approval: It is not declared by the authors.

Funding/Support: None. 


\section{References}

1. Catal F, Tayman C, Tonbul A, Akca H, Kara S, Tatli MM, et al. Mean platelet volume (MPV) may simply predict the severity of sepsis in preterm infants. Clin Lab. 2014;60(7):1193-200. doi: 10.7754/Clin.Lab.2013.130501. [PubMed: 25134389].

2. Alshorman A, Maghayreh M, Khriesat W, Swedan S. The effect of neonatal sepsis on platelet count and their indices. Jordan Med J. 2008;42(2):82-6.

3. Ahmad MS, Waheed A. Platelet counts, MPV and PDW in culture proven and probable neonatal sepsis and association of platelet counts with mortality rate.JColl Physicians Surg Pak. 2014;24(5):340-4. [PubMed: 24848393].

4. Ree I, Fustolo-Gunnink S, Bekker V, Te Pas A, Lopriore E. Thrombocytopenia in neonatal sepsis due to gram-negative versus gram-positive bacteria. Am J Perinatol. 2016;33(S 01). doi:10.1055/s-0036-1592387.

5. Ghanghoriya PK, Gedam DS. Neonatal septicemia and thrombocytopenia. Int J Med Res Rev. 2015;3(2):139-40.

6. Torkaman M, Afsharpaiman SH, Hoseini MI, Moradi M, Mazraati A, Amirsalari S, et al. Platelet count and neonatal sepsis: A high prevalence of Enterobacter spp. Singapore Med J. 2009;50(5):482-5. [PubMed: 19495516].

7. Bhat S, Naik S, Rafiq W, Tariq A. Incidence of thrombocytopenia and changes in various platelet parameters, in blood culture positive neonatal sepsis. Int J Pediat. 2015;3(4.1):757-66.

8. O'Connor TA, Ringer KM, Gaddis ML. Mean platelet volume during coagulase-negative staphylococcal sepsis in neonates. Am JClin Pathol. 1993;99(1):69-71. doi: 10.1093/ajcp/99.1.69. [PubMed: 8422020].

9. Manzoni P, Mostert M, Galletto P, Gastaldo L, Gallo E, Agriesti G, et al. Is thrombocytopenia suggestive of organism-specific response in neonatal sepsis? Pediatr Int. 2009;51(2):206-10. doi: 10.1111/j.1442200X.2008.02689.x. [PubMed: 19405917].

10. Ree IMC, Fustolo-Gunnink SF, Bekker V, Fijnvandraat KJ, Steggerda SI, Lopriore E. Thrombocytopenia in neonatal sepsis: Incidence, severity and risk factors. PLoS One. 2017;12(10). e0185581. doi: 10.1371/journal.pone.0185581. [PubMed: 28977011]. [PubMed Central: PMC5627935].
11. Ahmed F, Raj AY, Begum LN. Thrombocytopenia in late onset neonatal sepsis and its relationship with specific organism in a tertiary care hospital. Mymensingh Med J. 2017;26(4):900-5. [PubMed: 29208882].

12. Ahmad P, Kaith R, Gattoo I, Najar BA, Hussain SQ. Thrombocytopenia as a predictor of neonatal sepsis in very low birth weight babies and its correlation with specific organism involved. Ind J Neonatal Med Res. 2015;3(3):7-13.

13. Bhat MA, Bhat JI, Kawoosa MS, Ahmad SM, Ali SW. Organism-specific platelet response and factors affecting survival in thrombocytopenic very low birth weight babies with sepsis. J Perinatol. 2009;29(10):7028. doi:10.1038/jp.2009.72. [PubMed:19554015].

14. Shane AL, Stoll BJ. Recent developments and current issues in the epidemiology, diagnosis, and management of bacterial and fungal neonatal sepsis. Am J Perinatol. 2013;30(2):131-41. doi: 10.1055/s-00321333413. [PubMed: 23297182].

15. Karne TK, Joshi DD, Zile U, Patil S. Study of platelet count and platelet indices in neonatal sepsis in tertiary care institute. MVP J Med Sci. 2017;4(1):55. doi: 10.18311/mvpjms/0/v0/io/701.

16. Shalaby MM, Sobeih AA, Abdulghany WE, Behiry EG, Ismail YM, AbdEl-Aziz MA. Mean platelet volume and serum uric acid in neonatal sepsis: A case-control study. Ann Med Surg (Lond). 2017;20:97-102. doi: 10.1016/j.amsu.2017.06.015. [PubMed: 28761638]. [PubMed Central: PMC5522914]

17. Choudhary RR, Makwana M, Mourya HK, Dabi J, Gulati K. Evaluation of platelet and its indices as a marker of neonatal sepsis: A prospective case control study. Int J Cont Pediatr. 2018;5(5):1898. doi:10.18203/23493291.ijcp20183527.

18. Shaaban HA, Safwat N. Mean platelet volume in preterm: A predictor of early onset neonatal sepsis. J Matern Fetal Neonatal Med. 2018:1-6. doi: 10.1080/14767058.2018.1488161. [PubMed: 29886794].

19. Akarsu S, Taskin E, Kilic M, Ozdiller S, Gurgoze MK, Yilmaz E, et al The effects of different infectious organisms on platelet counts and platelet indices in neonates with sepsis: Is there an organism-specific response? J Trop Pediatr. 2005;51(6):388-91. doi:10.1093/tropej/fmi031. [PubMed: 16126807].

20. Guclu E, Durmaz Y, Karabay O. Effect of severe sepsis on platelet count and their indices. Afr Health Sci. 2013;13(2):333-8. doi: 10.4314/ahs.v13i2.19. [PubMed: 24235932]. [PubMed Central PMC3824485]. 